\title{
GLOBAL SOLUTIONS TO INVOLUTIVE SYSTEMS
}

\author{
A. P. BERGAMASCO, A. KIRILOV, W. V. L. NUNES, AND S. L. ZANI \\ (Communicated by Mei-Chi Shaw)
}

\begin{abstract}
In this work we consider a class of systems of two vector fields on the 3-torus associated to a closed smooth complex 1-form $c=a+i b$ with $b$ exact. Necessary conditions and sufficient conditions for this system to be globally solvable are provided in terms of the position of the global extrema and the connectedness of sublevel and superlevel sets of the primitives of $b$, together with arithmetical properties of the periods of $a$.
\end{abstract}

\section{INTRODUCTION}

Consider a smooth closed 1-form $c=c_{1} d t_{1}+c_{2} d t_{2}$ defined on the torus $\mathbb{T}^{2}$. Associated to this 1-form we have the following system of vector fields defined on $\mathbb{T}^{3} \doteq \mathbb{R}^{3} / 2 \pi \mathbb{Z}^{3}$ :

$$
L_{j}=\frac{\partial}{\partial t_{j}}+c_{j}(t) \frac{\partial}{\partial x}, \quad j=1,2,
$$

where $(t, x)=\left(t_{1}, t_{2}, x\right)$ are the coordinates in $\mathbb{T}^{3} \doteq \mathbb{T}_{\left(t_{1}, t_{2}\right)}^{2} \times \mathbb{T}_{x}^{1}$.

We set $a_{j}=\operatorname{Re} c_{j}, b_{j}=\operatorname{Im} c_{j}, j=1,2, a=a_{1} d t_{1}+a_{2} d t_{2}$ and $b \doteq b_{1} d t_{1}+b_{2} d t_{2}$.

We also define

$$
c_{10}=\frac{1}{2 \pi} \int_{0}^{2 \pi} c_{1}\left(\tau_{1}, 0\right) d \tau_{1} \quad \text { and } \quad c_{20}=\frac{1}{2 \pi} \int_{0}^{2 \pi} c_{2}\left(0, \tau_{2}\right) d \tau_{2},
$$

which are known as the periods of $c(t)$. Also, $a_{j 0} \doteq \operatorname{Re} c_{j 0}, b_{j 0} \doteq \operatorname{Im} c_{j 0}, j=1,2$.

Since $c(t)$ is closed, the system (1.1) defines an involutive structure in $\mathbb{T}^{3}$ (see $[8,10])$.

If $u \in \mathscr{D}^{\prime}\left(\mathbb{T}^{3}\right)$, and $f_{1}, f_{2} \in C^{\infty}\left(\mathbb{T}^{3}\right)$ are such that

$$
L_{1} u=f_{1} \quad \text { and } \quad L_{2} u=f_{2},
$$

then

$$
L_{1} f_{2}=L_{2} f_{1} .
$$

Taking Fourier series with respect to $x$ in the equations

$$
\frac{\partial u}{\partial t_{j}}+c_{j}(t) \frac{\partial u}{\partial x}=f_{j}, \quad j=1,2
$$

Received by the editors February 28, 2014 and, in revised form, September 11, 2014. 2010 Mathematics Subject Classification. Primary 35A05, 35N10, 58J10.

Key words and phrases. Global solvability, complex 1-form, periodic solutions.

The first author was supported in part by CNPq and FAPESP.

The third author was supported in part by FAPESP.

The fourth author was supported in part by CNPq and FAPESP. 
we are led to

$$
\frac{\partial \widehat{u}}{\partial t_{j}}(t, n)+i n c_{j}(t) \widehat{u}(t, n)=\widehat{f}_{j}(t, n), \quad n \in \mathbb{Z} .
$$

Let $C: \mathbb{R}^{2} \rightarrow \mathbb{C}$ be a primitive of the pull-back of $c$ via the universal covering $\Pi: \mathbb{R}^{2} \rightarrow \mathbb{T}^{2}$.

If $n \in \mathbb{Z}$ is such that $n\left(c_{10}, c_{20}\right) \in \mathbb{Z}^{2}$, then

$$
\mathbb{T}^{2} \ni t \mapsto e^{i n C(t)}
$$

is well defined, and we have

$$
\frac{\partial}{\partial t_{j}}\left(e^{i n C(t)} \widehat{u}(t, n)\right)=e^{i n C(t)} \widehat{f}_{j}(t, n),
$$

in other words, the 1-form

$$
e^{i n C(t)}\left(\widehat{f}_{1}(t, n) d t_{1}+\widehat{f}_{j}(t, n) d t_{2}\right)
$$

is exact, whenever $n\left(c_{10}, c_{20}\right) \in \mathbb{Z}^{2}$.

Let $\mathbb{E}$ be the set of pairs of functions $f_{1}, f_{2} \in C^{\infty}\left(\mathbb{T}^{3}\right)$, satisfying $L_{1} f_{2}=L_{2} f_{1}$, and such that

$$
e^{i n C(t)}\left(\widehat{f}_{1}(t, n) d t_{1}+\widehat{f}_{2}(t, n) d t_{2}\right)
$$

is exact, whenever $n\left(c_{10}, c_{20}\right) \in \mathbb{Z}^{2}$.

Definition 1.1. The system (1.1) is said to be globally solvable on $\mathbb{T}^{3}$ if for any $\left(f_{1}, f_{2}\right) \in \mathbb{E}$ there exists $u \in \mathscr{D}^{\prime}\left(\mathbb{T}^{3}\right)$ satisfying $L_{j} u=f_{j}, j=1,2$.

When $c$ is an exact 1-form, there exists, in particular, a global primitive of $b=\operatorname{Im} c$ defined on $\mathbb{T}^{2}$ and, in this case, the global solvability is equivalent to the connectedness of all superlevel and sublevel sets of such primitive. This result is due to Cardoso and Hounie and is a consequence of results in [9].

The real case $(b \equiv 0)$ was studied by the first author and Petronilho who showed in (7] that (1.1) is globally solvable if and only if the 1-form $a$ is either rational or non-Liouville.

When $a \equiv 0$ and $b$ is a closed, non-exact 1 -form on $\mathbb{T}^{2}$, one has that the global solvability of (1.1) is equivalent to the connectedness of all superlevel and sublevel sets of a global primitive of $\Pi^{*} b$, where $\Pi: \mathcal{T} \rightarrow \mathbb{T}^{2}$ is the minimal covering, that is, $\mathcal{T}=\mathbb{R}^{2}$ when the periods of $b$ are rationally independent and $\mathcal{T}=\mathbb{T}^{1} \times \mathbb{R}$ otherwise. The former result was proved in [3] and the latter one in [4].

In this work we study the case when $b$ is exact and $a \not \equiv 0$. In fact, our initial motivation was to seek an understanding of the influence of the real part of $c$ on the global solvability. That such an influence is far from negligible can be seen, for instance, in our main result; there, one can see that if one starts with a globally solvable system with $b$ exact and $a \equiv 0$, then, under a geometric assumption on the position of the global maximum of the primitives of $b$, the addition of a real part $a$ will turn the system into a non-solvable one except for a discrete and countable set of values of the periods of $a$. 
Here is our main result:

Theorem 1.2. Let $b(t)$ be exact and set $B(t)=\int_{0}^{t} b$. Suppose that there are two generators of the homology group of $\mathbb{T}^{2}$ which either do not intersect the level set $\left\{t \in \mathbb{T}^{2} ; B(t)=\max _{\tau \in \mathbb{T}^{2}} B(\tau)\right\}$ or else do not intersect the level set $\left\{t \in \mathbb{T}^{2} ; B(t)=\right.$ $\left.\min _{\tau \in \mathbb{T}^{2}} B(\tau)\right\}$. Then the system $\mathbb{L}$ given by

$$
L_{j}=\frac{\partial}{\partial t_{j}}+c_{j}(t) \frac{\partial}{\partial x}, \quad j=1,2,
$$

is globally solvable on $\mathbb{T}^{3}$ if and only if the following conditions are satisfied:

i. $\left(a_{10}, a_{20}\right) \in \mathbb{Z}^{2}$;

ii. for every $r \in \mathbb{R}$, the sublevels $\Omega_{r}=\left\{t \in \mathbb{T}^{2} ; B(t)<r\right\}$ and the superlevels $\Omega^{r}=\left\{t \in \mathbb{T}^{2} ; B(t)>r\right\}$ are connected.

It suffices to prove when the generators do not intersect the level set $\mathcal{F} \doteq\{t \in$ $\left.\mathbb{T}^{2} ; B(t)=\max _{\tau \in \mathbb{T}^{2}} B(\tau)\right\}$. Indeed, the remaining case follows from applying the change of variables $(t, x) \mapsto(t,-x)$.

Observe that if $b\left(t_{1}, t_{2}\right)=d_{t} \sin t_{2}=\cos t_{2} d t_{2}$, the condition about finding a couple of generators in $\mathbb{T}^{2}$ that do not intersect the lines $t_{2}=\pi / 2$ (maximum of $B$ ) or $t_{2}=3 \pi / 2$ (minimum of $B$ ) is not satisfied. Regarding this situation we have, with a construction more involved than the ones in Theorem 1.2, the following.

Theorem 1.3. Suppose $b(t)$ is exact and set $B(t)=\int_{0}^{t} b$. Suppose that we can find coordinates $\left(t_{1}, t_{2}, x\right)$ such that the following conditions are satisfied:

$i$. the maximum of the function $B(t) \doteq \int_{0}^{t} \Pi^{*} b$ on $[0,2 \pi]^{2}$ occurs on $\{0\} \times$ $(0,2 \pi)$ and does not occur on $[0,2 \pi] \times\{0\}$;

ii. $a_{10}$ is a Liouville number.

Then the system $\mathbb{L}$ is not globally solvable in $\mathbb{T}^{3}$.

We recall that an irrational number $\alpha$ is said to be a Liouville number if for each $N \in \mathbb{N}$ there are integers $p=p(N) \neq 0$ and $q=q(N)>1$ such that $|\alpha-p / q|<q^{-N}$.

Other articles dealing with global solvability and global hypoellipticity of systems of vector fields and directly related with this work are [2, 5, 6].

\section{Proof of Theorem 1.2}

Note that the real part of $c(t)$ can always be assumed to be constant. In fact, if $A: \mathbb{R}^{2} \rightarrow \mathbb{R}$ is a primitive of the pull-back of $a(t)$, then the map

$$
\sum_{n \in \mathbb{Z}} \hat{u}(t, n) e^{i n x} \longmapsto \sum_{n \in \mathbb{Z}} \hat{u}(t, n) e^{i n\left(A(t)-a_{10} t_{1}-a_{20} t_{2}\right)} e^{i n x}
$$

is an automorphism, $S$, of $\mathscr{D}^{\prime}\left(\mathbb{T}^{3}\right)$ and of $C^{\infty}\left(\mathbb{T}^{3}\right)$, which satisfies

$$
S L_{j} S^{-1}=\frac{\partial}{\partial t_{j}}+\left(a_{j 0}+i b_{j}(t)\right) \frac{\partial}{\partial x}, \quad j=1,2 .
$$

Furthermore, if, say, $a_{10} \in \mathbb{Z}$, then we can modify $S$ as

$$
\sum_{n \in \mathbb{Z}} \hat{u}(t, n) e^{i n x} \longmapsto \sum_{n \in \mathbb{Z}} \hat{u}(t, n) e^{i n\left(A(t)-a_{20} t_{2}\right)} e^{i n x}
$$

to obtain, for the first vector field $L_{1}$,

$$
S L_{1} S^{-1}=\frac{\partial}{\partial t_{1}}+i b_{1}(t) \frac{\partial}{\partial x} .
$$


Thus, the sufficiency of conditions $i$ and $i$ f follows from the result of Cardoso and Hounie in 9] after one performs the conjugation

$$
\sum_{n \in \mathbb{Z}} \hat{u}(t, n) e^{i n x} \longmapsto \sum_{n \in \mathbb{Z}} \hat{u}(t, n) e^{i n A(t)} e^{i n x} .
$$

In order to prove the necessity we may assume that $\left(a_{10}, a_{20}\right) \notin \mathbb{Z}^{2}$, for otherwise, we can conjugate and use once again the result in 9 .

Our next step will be to carry out the proof of necessity in the case when both $a_{10}$ and $a_{20}$ are irrational. After that we will indicate briefly how to take care of the remaining cases.

We may also assume that $a(t)=a_{10} d t_{1}+a_{20} d t_{2}$.

We want to choose a convenient function $h \in C^{\infty}\left(\mathbb{T}^{3}\right)$ in such a way that the equations

$$
L_{1} f_{2}=L_{2} f_{1}=h
$$

have smooth solutions, while

$$
L_{j} u=f_{j}, \quad j=1,2,
$$

have no solution in $\mathscr{D}^{\prime}\left(\mathbb{T}^{3}\right)$.

Taking partial Fourier series in $L_{1} f_{2}=L_{2} f_{1}=h$ we have

$$
\frac{\partial \widehat{f}_{2}}{\partial t_{1}}(t, n)+i n c_{1}(t) \widehat{f}_{2}(t, n)=\widehat{h}(t, n), \quad n \in \mathbb{Z},
$$

and

$$
\frac{\partial \widehat{f}_{1}}{\partial t_{2}}(t, n)+i n c_{2}(t) \widehat{f}_{1}(t, n)=\widehat{h}(t, n), \quad n \in \mathbb{Z} .
$$

Since $a_{10}$ and $a_{20}$ are both irrational numbers we have $i n a_{j 0} \notin i \mathbb{Z}, j=1,2$, for all $n \in \mathbb{Z}, n \neq 0$, and one can solve the equations above obtaining

$$
\begin{aligned}
& \widehat{f}_{1}(t, n)=d_{2 n} \int_{0}^{2 \pi} e^{-i n\left[C\left(t_{1}, t_{2}\right)-C\left(t_{1}, t_{2}-s_{2}\right)\right]} \widehat{h}\left(t_{1}, t_{2}-s_{2}, n\right) d s_{2}, \\
& \widehat{f}_{2}(t, n)=d_{1 n} \int_{0}^{2 \pi} e^{-i n\left[C\left(t_{1}, t_{2}\right)-C\left(t_{1}-s_{1}, t_{2}\right)\right]} \widehat{h}\left(t_{1}-s_{1}, t_{2}, n\right) d s_{1},
\end{aligned}
$$

where

$$
\begin{gathered}
d_{j n} \doteq\left(1-e^{2 \pi i n a_{j 0}}\right)^{-1}, j=1,2, \\
C(t) \doteq A(t)+i B(t), \\
d_{t} A(t)=a_{10} d t_{1}+a_{20} d t_{2}, \text { and } d_{t} B(t)=b_{1}(t) d t_{1}+b_{2}(t) d t_{2} .
\end{gathered}
$$

Thus, $h$ has to be chosen such that $\sum_{n} \widehat{f}_{j}(t, n) e^{i n x}$ is smooth, $j=1,2$. In other words, $\partial^{\alpha} \widehat{f}_{j}(t, n)$ has to decay rapidly in $n$, and uniformly in $t$, for each $\alpha \in \mathbb{Z}_{+}^{2}$.

If $L_{j} u=f_{j}, j=1,2$, happens to have a solution $u$, then, for $n \neq 0$, its partial Fourier coefficients are determined by

$$
\widehat{u}(t, n)=d_{1 n} d_{2 n} \int_{0}^{2 \pi} \int_{0}^{2 \pi} e^{-i n[C(t)-C(t-s)]} \widehat{h}(t-s, n) d s_{1} d s_{2} .
$$

If we choose $\hat{h}(t, 0)=\hat{h}(t, 0)=0$, then we can take $\hat{f}_{1}(t, 0)=\hat{f}_{2}(t, 0)=0$ which implies that $\left(f_{1}, f_{2}\right) \in \mathbb{E}$.

We are done if $h$ has the additional property that the right-hand side of (2.5) does not grow slowly in $n$ for some $t$. 
We may regard $B$ as a function defined in $\mathbb{R}^{2}$ that is $2 \pi$-periodic in each variable. Furthermore, it follows from the assumptions in Theorem 1.2 that this identification can be achieved in such a way that the maximum of $B$ does not occur on the boundary of the square $[0,2 \pi]^{2}$.

Let $M$ be the maximum of $B$ in $[0,2 \pi]^{2}$ and take $t^{*}=\left(t_{1}^{*}, t_{2}^{*}\right) \in(0,2 \pi)^{2}$ such that $B\left(t^{*}\right)=M$. Obviously, we can assume that $B(0)=A\left(t^{*}\right)=0<M$.

Now setting

$$
M^{\prime} \doteq \max _{t_{1} \in[0,2 \pi]} B\left(t_{1}, 0\right) \text { and } M^{\prime \prime} \doteq \max _{t_{2} \in[0,2 \pi]} B\left(0, t_{2}\right)
$$

we have

$$
0 \leq M^{\prime}, M^{\prime \prime}<M
$$

Let $\chi_{\delta} \in C_{c}^{\infty}\left(\mathbb{R}^{2}\right)$ be a function such that $0 \leq \chi_{\delta} \leq 1$,

$$
\chi_{\delta}(t)= \begin{cases}1, & \text { if } t \in Q_{\delta}, \\ 0, & \text { if } t \in \mathbb{R}^{2} \backslash Q_{2 \delta},\end{cases}
$$

where $Q_{\delta} \doteq\left\{\tau \in \mathbb{R}^{2} ;\left|\tau_{j}\right| \leq \delta, j=1,2\right\}$, and $0<\delta<1$ is small enough such that

$$
|B(t)| \leq \frac{M}{2}, t \in Q_{2 \delta}
$$

Setting

$$
Q^{*} \doteq-\frac{1}{2} t^{*}+[0,2 \pi]^{2}
$$

consider the $2 \pi$-periodic real smooth functions $q$ and $r$ defined on $\mathbb{R}^{2}$ such that

$$
\begin{aligned}
& q(t) \doteq\left(|t|^{2}-1\right) \cdot \chi_{\delta}(t)+1, \\
& r(t) \doteq A(t) \cdot \chi_{\delta}(t),
\end{aligned}
$$

for every $t \in Q^{*}$.

Decreasing $\delta>0$, if necessary, we may assume that $Q_{2 \delta} \subset \operatorname{int}\left(Q^{*}\right)$.

For $t \in \mathbb{R}^{2}$ and $n \in \mathbb{Z}$, we define

$$
\hat{h}(t, n) \doteq \begin{cases}c_{n} e^{-n[M-B(t)+K q(t)+i r(t)]}, & \text { if } n \geq 1, \\ 0, & \text { if } n \leq 0,\end{cases}
$$

where $K>0$ and the sequence $\left\{c_{n}\right\}_{n \in \mathbb{N}}$ will both be chosen later on, and the function $h$ is given by

$$
h(t, x) \doteq \sum_{n \in \mathbb{Z}} \hat{h}(t, n) e^{i n x},(t, x) \in \mathbb{R}^{3} .
$$

For each $t \in \mathbb{R}^{2}$ and $n \geq 1$, by substituting the coefficients $\hat{h}(t, n)$ in the formulas (2.3), (2.4) and (2.5), we obtain

$$
\begin{aligned}
& \hat{f}_{1}(t, n)=c_{n} d_{2 n} \int_{0}^{2 \pi} e^{-n \phi\left(t_{1}, t_{2}, 0, s_{2}\right)} d s_{2}, \\
& \hat{f}_{2}(t, n)=c_{n} d_{1 n} \int_{0}^{2 \pi} e^{-n \phi\left(t_{1}, t_{2}, s_{1}, 0\right)} d s_{1}, \\
& \hat{u}(t, n)=c_{n} d_{1 n} d_{2 n} \int_{0}^{2 \pi} \int_{0}^{2 \pi} e^{-n \phi(t, s)} d s_{1} d s_{2},
\end{aligned}
$$


where

$$
\phi(t, s) \doteq i[C(t)-C(t-s)]+M-B(t-s)+K q(t-s)+i r(t-s)
$$

for $(t, s) \in \mathbb{R}^{2} \times \mathbb{R}^{2}$, and we set

$$
\begin{aligned}
\varphi(t, s) & \doteq-\operatorname{Re}[\phi(t, s)]=B(t)-M-K q(t-s) \\
\psi(t, s) & \doteq \operatorname{Im}[\phi(t, s)]=A(t)-A(t-s)+r(t-s) .
\end{aligned}
$$

Since for each $n \in \mathbb{N}, \hat{h}(\cdot, n), \hat{f}_{1}(\cdot, n), \hat{f}_{2}(\cdot, n)$ and $\hat{u}(\cdot, n)$ are $2 \pi$-periodic smooth functions in $\mathbb{R}^{2}$, it is enough to study their behavior in a square of sidelength $2 \pi$, in particular, in $Q^{*}$ given by (2.8).

We have

Proposition 2.1. Let $\left\{c_{n}\right\}_{n \in \mathbb{N}}$ be a sequence such that

$$
\left\{c_{n} e^{-n \frac{M}{2}}\right\}_{n \in \mathbb{N}} \in \mathscr{S}(\mathbb{N}) .
$$

Then for all sufficiently large $K>0$ we have $h \in C^{\infty}\left(\mathbb{T}^{3}\right)$.

Proof. We choose $K>1$ such that $K \geq M / 2$.

We observe that if $t \in Q^{*} \backslash Q_{2 \delta}$, then $\chi_{\delta} \equiv 0$ which implies $q(t)=1$ and $M-B(t)+K q(t)=M-B(t)+K \geqslant M / 2$. Now, if $t \in Q_{\delta}$, then $\chi_{\delta} \equiv 1$ and $|B(t)| \leqslant M / 2$, by (2.7), thus $M-B(t)+K q(t)=M-B(t)+K|t|^{2} \geqslant M / 2$. Finally if $t \in Q_{2 \delta} \backslash Q_{\delta}$, then $|B(t)| \leqslant M / 2$ and $q(t) \geqslant 0$ which implies $M-B(t)+K q(t) \geqslant$ $M-B(t) \geqslant M / 2$.

Thus $M-B(t)+K q(t) \geqslant M / 2$, for all $t \in Q^{*}$, which implies

$$
|\hat{h}(t, n)|=\left|c_{n}\right| e^{-n[M-B(t)+K q(t)]}<\left|c_{n}\right| e^{-n M / 2},
$$

for all $n \in \mathbb{N}$ and $t \in Q^{*}$.

For $\alpha, \beta \in \mathbb{Z}_{+}$, we have $\partial^{\alpha+\beta} \hat{h}(t, n)=\hat{h}(t, n) P(t, n, \alpha, \beta)$, where $P$ satisfies $|P(t, n, \alpha, \beta)| \leq C_{\alpha, \beta} n^{\alpha+\beta}, n \in \mathbb{N}$ and $t \in Q^{*}$, which implies

$$
\left|\partial^{(\alpha, \beta)} \hat{h}(t, n)\right| \leq C_{\alpha, \beta} n^{\alpha+\beta}\left|c_{n}\right| e^{-n M / 2},
$$

for all $t \in Q^{*}$, and $n \in \mathbb{N}$. Since $\left\{c_{n} e^{-n \frac{M}{2}}\right\}_{n \in \mathbb{N}} \in \mathscr{S}(\mathbb{N})$, it follows that $h \in$ $C^{\infty}\left(\mathbb{T}^{3}\right)$.

We also have

Proposition 2.2. Let $\left\{c_{n}\right\}_{n \in \mathbb{N}}$ be a sequence such that

$$
\left\{c_{n} d_{2 n} e^{n \frac{M^{\prime \prime}-M}{2}}\right\}_{n \in \mathbb{N}} \in \mathscr{S}(\mathbb{N}) .
$$

Then, for all sufficiently large $K>0$,

$$
f_{1}(t, x)=\sum_{n=1}^{\infty} \hat{f}_{1}(t, n) e^{i n x}
$$

with $\hat{f}_{1}(t, n)$ given by (2.13) defines a smooth function.

Proof. Let $\varphi_{0}\left(t_{1}, t_{2}, s_{2}\right) \doteq \varphi\left(t_{1}, t_{2}, 0, s_{2}\right),\left(t_{1}, t_{2}, s_{2}\right) \in Q^{*} \times[0,2 \pi]$.

From (2.13) we obtain

$$
\left|\hat{f}_{1}(t, n)\right| \leq 2 \pi\left|c_{n} d_{2 n}\right| \sup _{s_{2} \in[0,2 \pi]} e^{-n \varphi_{0}\left(t_{1}, t_{2}, s_{2}\right)}
$$

where $\varphi_{0}\left(t_{1}, t_{2}, s_{2}\right)=B\left(t_{1}, t_{2}\right)-M-K q\left(t_{1}, t_{2}-s_{2}\right)$. 
It is useful to find all points $\left(t_{1}, t_{2}, s_{2}\right) \in Q^{*} \times[0,2 \pi]$ such that $q\left(t_{1}, t_{2}-s_{2}\right)=0$ because at those points, $K$ plays no role in the decay of $\hat{f}_{1}(t, n)$.

We have $q\left(t_{1}, t_{2}-s_{2}\right)=0$, with $\left(t_{1}, t_{2}, s_{2}\right) \in Q^{*} \times[0,2 \pi]$, if and only if $\left(t_{1}, t_{2}, s_{2}\right)$ is in the set

$$
X \doteq\left\{\left(0, t_{2}, t_{2}\right) ; 0 \leq t_{2} \leq 2 \pi-t_{2}^{*} / 2\right\} \cup\left\{\left(0, t_{2}, t_{2}+2 \pi\right) ;-t_{2}^{*} / 2 \leq t_{2} \leq 0\right\},
$$

and on such points

$$
\varphi_{0}\left(t_{1}, t_{2}, s_{2}\right)=B\left(0, t_{2}\right)-M \leq M^{\prime \prime}-M .
$$

Since $X$ is a compact set, there exists an open neighborhood $\mathcal{U}$ of $X$ such that

$$
\varphi_{0}\left(t_{1}, t_{2}, s_{2}\right)<\frac{1}{2}\left(M^{\prime \prime}-M\right), \text { for }\left(t_{1}, t_{2}, s_{2}\right) \in \mathcal{U} \text {. }
$$

Let $\eta>0$ be the global minimum of $\left(t_{1}, t_{2}, s_{2}\right) \mapsto q\left(t_{1}, t_{2}-s_{2}\right)$ on the compact set $F \doteq\left(Q^{*} \times[0,2 \pi]\right) \backslash \mathcal{U}$. Then

$$
\varphi_{0}\left(t_{1}, t_{2}, s_{2}\right) \leq-K q\left(t_{1}, t_{2}-s_{2}\right) \leq-K \eta, \forall\left(t_{1}, t_{2}, s_{2}\right) \in F .
$$

We may increase $K>1$, if necessary, so that $-K \eta<\frac{1}{2}\left(M^{\prime \prime}-M\right)$, and from (2.6), we have

$$
\varphi_{0}\left(t_{1}, t_{2}, s_{2}\right)<\frac{1}{2}\left(M^{\prime \prime}-M\right)<0,\left(t_{1}, t_{2}, s_{2}\right) \in Q^{*} \times[0,2 \pi] .
$$

Therefore,

$$
\left|\hat{f}_{1}(t, n)\right| \leq\left|c_{n} d_{2 n}\right| 2 \pi e^{n\left(M^{\prime \prime}-M\right) / 2} .
$$

Finally, for each $\alpha, \beta \in \mathbb{Z}^{+}$, we have

$$
\left|\partial^{(\alpha, \beta)} \hat{f}_{1}(t, n)\right| \leq\left|c_{n} d_{2 n}\right| \int_{0}^{2 \pi} e^{n \varphi_{0}\left(t_{1}, t_{2}, s_{2}\right)}|P(t, n, \alpha, \beta)| d s_{2},
$$

where $P(t, n, \alpha, \beta)$ satisfies $|P(t, n, \alpha, \beta)| \leq C_{\alpha, \beta} n^{\alpha+\beta}, \forall n \in \mathbb{N}$ and $t \in Q^{*}$, for some $C_{\alpha, \beta}>0$, which implies

$$
\begin{aligned}
\left|\partial^{(\alpha, \beta)} \hat{f}_{1}(t, n)\right| & \leq\left|c_{n} d_{2 n}\right| C_{\alpha, \beta} n^{\alpha+\beta} \int_{0}^{2 \pi} e^{n \varphi_{0}\left(t_{1}, t_{2}, s_{2}\right)} d s_{2} \\
& \leq 2 \pi\left|c_{n} d_{2 n}\right| C_{\alpha, \beta} n^{\alpha+\beta} e^{n\left(M^{\prime \prime}-M\right) / 2}, \forall n \in \mathbb{N} \text { and } t \in Q^{*}
\end{aligned}
$$

Since $\left\{c_{n}\right\}_{n \in \mathbb{N}}$ satisfies (2.21), it follows that $f_{1} \in C^{\infty}\left(\mathbb{T}^{3}\right)$.

We have a similar result for $f_{2}$, the proof of which will be omitted.

Proposition 2.3. Let $\left\{c_{n}\right\}_{n \in \mathbb{N}}$ be a sequence such that

$$
\left\{c_{n} d_{1 n} e^{n \frac{M^{\prime}-M}{2}}\right\}_{n \in \mathbb{N}} \in \mathscr{S}(\mathbb{N}) .
$$

Then, for all sufficiently large $K>0$,

$$
f_{2}(t, x)=\sum_{n=1}^{\infty} \hat{f}_{2}(t, n) e^{i n x}
$$

with $\hat{f}_{2}(t, n)$ given by (2.14) defines a smooth function.

The last step is to show that for some $t \in \mathbb{T}^{2}$, the sequence $\{\hat{u}(t, n)\}_{n \in \mathbb{N}}$, given by (2.15), does not correspond to a distribution on the torus $\mathbb{T}^{3}$. 
Proposition 2.4. Let $\left\{c_{n}\right\}_{n \in \mathbb{N}}$ be a sequence such that

$$
\left\{c_{n} d_{1 n} d_{2 n}\right\}_{n \in \mathbb{N}} \notin \mathscr{S}^{\prime}(\mathbb{N}) .
$$

Then, for all $K>0$, the sequence on the right-hand side of (2.15) does not correspond to the partial Fourier coefficients of any distribution on the torus $\mathbb{T}^{3}$.

Proof. By (2.15) we have

$$
\begin{aligned}
\hat{u}\left(t^{*}, n\right) & =c_{n} d_{1 n} d_{2 n} \int_{0}^{2 \pi} \int_{0}^{2 \pi} e^{-n \phi\left(t^{*}, s\right)} d s_{1} d s_{2} \\
& =c_{n} d_{1 n} d_{2 n} \int_{0}^{2 \pi} \int_{0}^{2 \pi} e^{n(\varphi-i \psi)\left(t^{*}, s\right)} d s_{1} d s_{2},
\end{aligned}
$$

where

$$
\begin{aligned}
& \varphi\left(t^{*}, s\right)=B\left(t^{*}\right)-M-K q\left(t^{*}-s\right),=-K q\left(t^{*}-s\right), \\
& \psi\left(t^{*}, s\right)=A\left(t^{*}\right)-A\left(t^{*}-s\right)+r\left(t^{*}-s\right) .
\end{aligned}
$$

We rewrite $\hat{u}\left(t^{*}, n\right)$ as follows:

$$
\hat{u}\left(t^{*}, n\right)=c_{n} d_{1 n} d_{2 n}\left(I_{n}+J_{n}\right),
$$

where

$$
I_{n}=\iint_{\left|t^{*}-s\right|<\delta} e^{-n \phi\left(t^{*}, s\right)} d s_{1} d s_{2} \quad \text { and } \quad J_{n} \doteq \iint_{\left|t^{*}-s\right| \geq \delta} e^{-n \phi\left(t^{*}, s\right)} d s_{1} d s_{2} .
$$

We now study the behavior of the sequences $\left\{I_{n}\right\}_{n \in \mathbb{N}}$ and $\left\{J_{n}\right\}_{n \in \mathbb{N}}$.

Lemma 2.5. The sequence $\left\{J_{n}\right\}_{n \in \mathbb{N}}$ decays exponentially when $n \rightarrow \infty$.

Proof. If $\left|t^{*}-s\right| \geq \delta$, then we have $\varphi\left(t^{*}, s\right)=-K q\left(t^{*}-s\right) \leq-K \delta^{2}$, which implies

$$
0 \leq\left|\iint_{\left|t^{*}-s\right| \geq \delta} e^{-n \phi\left(t^{*}, s\right)} d s_{1} d s_{2}\right| \leq \iint_{\left|t^{*}-s\right| \geq \delta} e^{n \varphi\left(t^{*}, s\right)} d s_{1} d s_{2} \leq 4 \pi^{2} e^{-n K \delta^{2}},
$$

for all $n \in \mathbb{N}$, and thus $\left\{J_{n}\right\}_{n \in \mathbb{N}}$ decays exponentially when $n \rightarrow \infty$.

Lemma 2.6. There exists a constant $C>0$ such that

$$
C \frac{1}{n K} \leq\left|I_{n}\right| \leq \pi \frac{1}{n K}, \quad n \in \mathbb{N} .
$$

Proof. Note that if $\left|t^{*}-s\right|<\delta$, then $\chi_{\delta}\left(t^{*}-s\right)=1$, which implies

$$
\begin{aligned}
\phi\left(t^{*}, s\right) & =-K q\left(t^{*}-s\right)-i\left(A\left(t^{*}\right)-A\left(t^{*}-s\right)+r\left(t^{*}-s\right)\right) \\
& =-K\left|t^{*}-s\right|^{2}-i A\left(t^{*}\right)=-K\left|t^{*}-s\right|^{2},
\end{aligned}
$$

thus, we have

$$
\left|I_{n}\right|=\iint_{\left|t^{*}-s\right|<\delta} e^{n K\left|t^{*}-s\right|^{2}} d s_{1} d s_{2}
$$

Making the change of variable $\sigma \doteq \sqrt{n K}\left(t^{*}-s\right)$ in the above integral we obtain

$$
\left|I_{n}\right|=\frac{1}{n K} \iint_{|\sigma|<\delta \sqrt{n K}} e^{-|\sigma|^{2}} d \sigma_{1} d \sigma_{2}
$$

which implies that $\left|I_{n}\right|=C(n) / n K$ where $C(n) \doteq \iint_{|\sigma|<\delta \sqrt{n K}} e^{-|\sigma|^{2}} d \sigma_{1} d \sigma_{2}$. 
We note that $\{C(n)\}_{n \in \mathbb{N}}$ is an increasing sequence and

$$
C(1) \leq C(n)=\iint_{|\sigma|<\delta \sqrt{n K}} e^{-|\sigma|^{2}} d \sigma_{1} d \sigma_{2} \leq \iint_{\mathbb{R}^{2}} e^{-|\sigma|^{2}} d \sigma_{1} d \sigma_{2}=\pi .
$$

Therefore, $C(1) \frac{1}{n K} \leq\left|I_{n}\right| \leq \pi \frac{1}{n K}$, for all $n \in \mathbb{N}$.

Now we resume the proof of Proposition 2.4. By Lemmas 2.5] and 2.6 we have

$$
C(1) \frac{1}{n K} c_{n} d_{1 n} d_{2 n} \leq u\left(t^{*}, n\right), \quad n \in \mathbb{N} .
$$

Since $\left\{c_{n}\right\}_{n \in \mathbb{N}}$ satisfies (2.24), then $\left\{\hat{u}\left(t^{*}, n\right)\right\}_{n \in \mathbb{N}}$ is not slowly increasing, completing the proof of Proposition 2.4 .

We now present a sequence $\left\{c_{n}\right\}_{n \in \mathbb{N}}$ satisfying the conditions required in Propositions 2.1 through 2.4

For this, consider the sequence $\left\{c_{n}\right\}_{n \in \mathbb{N}}$ given by

$$
c_{n} \doteq \frac{e^{n L}}{d_{1 n} d_{2 n}}, \quad n \in \mathbb{N},
$$

where $L \doteq \min \left\{\left(M-M^{\prime}\right) / 4,\left(M-M^{\prime \prime}\right) / 4\right\}>0$.

Note that, for any $n \in \mathbb{N}$ :

$$
\begin{aligned}
& i . \quad\left|c_{n}\right| e^{-n \frac{M}{2}} \leq \frac{e^{n L}}{\left|d_{1 n} d_{2 n}\right|} e^{-n \frac{M}{2}} \leq 4 e^{n\left[\frac{M}{4}-\frac{M}{2}\right]}=4 e^{-n \frac{M}{4}} ; \\
& \text { ii. } \quad\left|c_{n} d_{2 n}\right| e^{-n \frac{M-M^{\prime \prime}}{2}} \leq \frac{e^{n L}}{\left|d_{1 n} d_{2 n}\right|}\left|d_{2 n}\right| e^{-n \frac{M-M^{\prime \prime}}{2}} \leq 2 e^{-n \frac{M-M^{\prime \prime}}{4}} \\
& \text { iii. } \quad\left|c_{n} d_{1 n}\right| e^{-n \frac{M-M^{\prime}}{2}} \leq \frac{e^{n L}}{\left|d_{1 n} d_{2 n}\right|}\left|d_{1 n}\right| e^{-n \frac{M-M^{\prime}}{2}} \leq 2 e^{-n \frac{M-M^{\prime}}{4}} \\
& \text { iv. } \quad\left|c_{n} d_{1 n} d_{2 n}\right|=\frac{e^{n L}}{\left|d_{1 n}\right|\left|d_{2 n}\right|}\left|d_{1 n}\right|\left|d_{2 n}\right|=e^{n L} .
\end{aligned}
$$

This finishes the proof of Theorem 1.2 in the case when $a_{10}$ and $a_{20}$ are irrational numbers.

In the case that $a_{10}$ is irrational and $a_{20}$ is rational, one can make a change of variables in $\mathbb{T}^{2}$ that preserves the assumption on $\mathcal{F}$ to obtain that both $a_{10}$ and $a_{20}$ are irrational numbers. More precisely, if $\sigma_{1}$ and $\sigma_{2}$ are the generators as in Theorem 1.2 then the new generators will be homologous to $\sigma_{1}$ and $\sigma_{1}+\sigma_{2}$, respectively. Similarly, if $a_{20}$ is irrational and $a_{10}$ is rational.

In the case when $a_{10}, a_{20} \in \mathbb{Q} \backslash \mathbb{Z}$, the proof can be adapted by setting $\hat{h}(t, n)=0$ for all the frequencies $n \in \mathbb{N}$ where $n a_{10}$ or $n a_{20}$ are integers.

In the case, say, when $a_{10} \in \mathbb{Z}$ and, hence, $a_{20} \notin \mathbb{Z}$, as before, it is possible to find new coordinates in $\mathbb{T}^{2}$ such that the corresponding $a_{10}$ and $a_{20}$ are both non-integral numbers.

The proof of Theorem 1.2 is complete. 


\section{Proof of Theorem 1.3}

Since $a_{10}$ is a Liouville number, it follows that there exists an increasing sequence $\left\{n_{k}\right\}_{k \in \mathbb{N}}, n_{k} \in \mathbb{N}$, such that

$$
\left|d_{1 n_{k}}\right|=\left|\frac{1}{1-e^{-2 \pi i n_{k} a_{10}}}\right|>n_{k}^{k} .
$$

First we address the case when $a_{20}$ is an irrational number. We use the same notation as in the previous section.

By hypothesis $i$, the maximum of $B$ on the square $[0,2 \pi]^{2}$ is attained at a point $t^{*}=\left(0, t_{2}^{*}\right)$ with $0<t_{2}^{*}<2 \pi$.

By hypothesis $i i$, we have

$$
0 \leq M^{\prime}<M=M^{\prime \prime}
$$

As before we will define a smooth function $h$ such that there exists a pair of smooth functions $\left(f_{1}, f_{2}\right) \in \mathbb{E}$ satisfying $L_{1} f_{2}=L_{2} f_{1}=h$, but the equations $L_{j} u=f_{j}, j=1,2$, have no solution in $\mathscr{D}^{\prime}$.

Let us fix a sequence $\left\{n_{k}\right\}_{k \in \mathbb{N}}$ such that (3.1) holds, and for each $n \in \mathbb{N}$ and $t \in \mathbb{R}^{2}$ define

$$
\hat{h}(t, n) \doteq \begin{cases}c_{n_{k}} e^{-n_{k}[M-B(t)+K q(t)+i r(t)]}, & n=n_{k} \text { for some } k \geq 1, \\ 0, & \text { otherwise }\end{cases}
$$

where the value of $K>0$ and the sequence $\left\{c_{n_{k}}\right\}_{k \in \mathbb{N}}$ will be chosen later. Let $h$ be the function given by (2.12).

The proofs of the results below are similar to the ones given in the previous section and will be omitted.

Proposition 3.1. Let $\left\{c_{n_{k}}\right\}_{k \in \mathbb{N}}$ be a sequence that satisfies

$$
\left\{c_{n_{k}} e^{-n_{k} \frac{M}{2}}\right\}_{k \in \mathbb{N}} \in \mathscr{S}(\mathbb{N}) .
$$

Then $h \in C^{\infty}\left(\mathbb{T}^{3}\right)$.

Proposition 3.2. Let $\left\{c_{n_{k}}\right\}_{k \in \mathbb{N}}$ be a sequence that satisfies

$$
\left\{c_{n_{k}} d_{2 n_{k}}\right\}_{k \in \mathbb{N}} \in \mathscr{S}(\mathbb{N}) .
$$

Then, for all sufficiently large $K>0$,

$$
f_{1}(t, x)=\sum_{n=1}^{\infty} \hat{f}_{1}(t, n) e^{i n x}
$$

with $\hat{f}_{1}(t, n)$ given by (2.13), and $\hat{h}(t, n)$ defined by (3.3), is a smooth function.

Proposition 3.3. Let $\left\{c_{n_{k}}\right\}_{k \in \mathbb{N}}$ be a real sequence such that

$$
\left\{c_{n_{k}} d_{1 n_{k}} e^{n_{k} \frac{M^{\prime}-M}{2}}\right\}_{k \in \mathbb{N}} \in \mathscr{S}(\mathbb{N}) .
$$

Then, for all sufficiently large $K>0$,

$$
f_{2}(t, x)=\sum_{n=1}^{\infty} \hat{f}_{2}(t, n) e^{i n x}
$$

with $\hat{f}_{2}(t, n)$ given by (2.14), with $\hat{h}(t, n)$ defined by (3.3), is a smooth function. 
Proposition 3.4. Let $\left\{c_{n_{k}}\right\}_{k \in \mathbb{N}}$ be a sequence such that

$$
\left\{c_{n_{k}} d_{1 n_{k}} d_{2 n_{k}}\right\}_{k \in \mathbb{N}} \notin \mathscr{S}^{\prime}(\mathbb{N}) .
$$

Then, for all $K>0$, the sequence on the right-hand side of (2.15) does not correspond to the partial Fourier coefficients of any distribution on the torus $\mathbb{T}^{3}$.

In order to finish the proof of Theorem 1.3. we need to present a sequence $\left\{c_{n_{k}}\right\}$ satisfying the conditions required in Propositions 3.1 through 3.4

Now, consider the following sets:

$$
\mathcal{I}_{1} \doteq\left\{n_{k} ;\left|d_{1 n_{k}}\right| \geqslant e^{n_{k} \frac{M-M^{\prime}}{2}}\right\} \quad \text { and } \quad \mathcal{I}_{2} \doteq\left\{n_{k} ;\left|d_{1 n_{k}}\right|<e^{n_{k} \frac{M-M^{\prime}}{2}}\right\} .
$$

We observe that either $\mathcal{I}_{1}$ or $\mathcal{I}_{2}$ is an infinite set.

a. If $\mathcal{I}_{1}$ is an infinite set, we take

$$
c_{n_{k}} \doteq \begin{cases}\left|d_{1 n_{k}} d_{2 n_{k}}\right|^{-1} e^{n_{k}\left(M-M^{\prime}\right) / 4}, & n_{k} \in \mathcal{I}_{1}, \\ 0, & \text { otherwise }\end{cases}
$$

b. Otherwise, $\mathcal{I}_{2}$ is an infinite set, and we take

$$
c_{n_{k}} \doteq \begin{cases}\left|d_{1 n_{k}}\right|^{-1 / 2}\left|d_{2 n_{k}}\right|^{-1}, & n_{k} \in \mathcal{I}_{2}, \\ 0, & \text { otherwise }\end{cases}
$$

In case (a), for any $k \in \mathbb{N}$, we have:

$$
\begin{aligned}
& a_{1} . \quad\left|c_{n_{k}} e^{-n_{k} M / 2}\right| \leq\left|d_{1 n_{k}} d_{2 n_{k}}\right|^{-1} e^{n_{k}\left(M-M^{\prime}\right) / 4} e^{-n_{k} M / 2} \\
& \leq 4 e^{-n_{k}\left(M+M^{\prime}\right) / 4} \\
& a_{2} . \quad\left|c_{n_{k}} d_{2 n_{k}}\right| \leq\left|d_{2 n_{k}}\right|\left|d_{1 n_{k}} d_{2 n_{k}}\right|^{-1} e^{n_{k}\left(M-M^{\prime}\right) / 4} \\
& \leq e^{-n_{k}\left(M-M^{\prime}\right) / 2} e^{n_{k}\left(M-M^{\prime}\right) / 4} \\
& \leq e^{-n_{k}\left(M-M^{\prime}\right) / 4} \\
& a_{3} . \quad\left|c_{n_{k}} d_{1 n_{k}} e^{-n_{k}\left(M-M^{\prime}\right) / 2}\right| \leq\left|d_{1 n_{k}} d_{2 n_{k}}\right|^{-1} e^{n_{k}\left(M-M^{\prime}\right) / 4}\left|d_{1 n_{k}}\right| e^{-n_{k}\left(M-M^{\prime}\right) / 2} \\
& \leq 2 e^{-n_{k}\left(M-M^{\prime}\right) / 4} \\
& \text { a. } \quad\left|c_{n_{k}} d_{1 n_{k}} d_{2 n_{k}}\right|=\left|d_{1 n_{k}} d_{2 n_{k}}\right|^{-1} e^{n_{k}\left(M-M^{\prime}\right) / 4}\left|d_{1 n_{k}}\right|\left|d_{2 n_{k}}\right| \\
& =e^{n_{k}\left(M-M^{\prime}\right) / 4} \text {, for } n_{k} \in \mathcal{I}_{1} \text {. }
\end{aligned}
$$

In case (b), for any $k \in \mathbb{N}$, we have:

$$
\begin{aligned}
& b_{1} . \quad\left|c_{n_{k}} e^{-n_{k} M / 2}\right| \leq\left|d_{1 n_{k}}\right|^{-1 / 2}\left|d_{2 n_{k}}\right|^{-1} e^{-n_{k} M / 2} \\
& \leq 2 \sqrt{2} e^{-n_{k} M / 2} \\
& b_{2} . \quad\left|c_{n_{k}} d_{2 n_{k}}\right|=\left|d_{1 n_{k}}\right|^{-1 / 2}\left|d_{2 n_{k}}\right|^{-1}\left|d_{2 n_{k}}\right| \\
& \begin{aligned}
b_{3} . \quad\left|c_{n_{k}} d_{1 n_{k}} e^{n_{k}\left(M^{\prime}-M\right) / 2}\right| & \leq n_{k}^{-k / 2} ; \\
& \leq\left|d_{1 n_{k}}\right|^{-1 / 2}\left|d_{2 n_{k}}\right|^{-1}\left|d_{1 n_{k}}\right| e^{n_{k}\left(M^{\prime}-M\right) / 2}
\end{aligned} \\
& \leq 2\left|d_{1 n_{k}}\right|^{1 / 2} e^{n_{k}\left(M^{\prime}-M\right) / 2} \\
& \leq e^{n_{k}\left(M-M^{\prime}\right) / 4} e^{n_{k}\left(M^{\prime}-M\right) / 2}=e^{-n_{k}\left(M-M^{\prime}\right) / 4} \text {; } \\
& \text { b. } \quad\left|c_{n_{k}} d_{1 n_{k}} d_{2 n_{k}}\right|=\left|d_{1 n_{k}}\right|^{-1 / 2}\left|d_{2 n_{k}}\right|^{-1}\left|d_{1 n_{k}} d_{2 n_{k}}\right| \\
& =\left|d_{1 n_{k}}\right|^{1 / 2} \geq n_{k}^{k / 2} \text {, for } n_{k} \in \mathcal{I}_{2} \text {. }
\end{aligned}
$$

This finishes the proof of Theorem 1.3 in the case when $a_{20}$ is an irrational number.

When $a_{20} \in \mathbb{Q}$, one can make a change of variables in $\mathbb{T}^{2}$ that preserves the assumption on $\mathcal{F}$ to obtain that $a_{20}$ is an irrational number, and $a_{10}$ is the same Liouville number. 


\section{REFERENCES}

[1] Adalberto P. Bergamasco, Remarks about global analytic hypoellipticity, Trans. Amer. Math. Soc. 351 (1999), no. 10, 4113-4126, DOI 10.1090/S0002-9947-99-02299-0. MR.1603878 (99m:35032)

[2] Adalberto P. Bergamasco, Cleber de Medeira, and Sérgio Luís Zani, Globally solvable systems of complex vector fields, J. Differential Equations 252 (2012), no. 8, 4598-4623, DOI 10.1016/j.jde.2012.01.007. MR2881049(2012m:35364)

[3] Adalberto P. Bergamasco and Alexandre Kirilov, Global solvability for a class of overdetermined systems, J. Funct. Anal. 252 (2007), no. 2, 603-629, DOI 10.1016/j.jfa.2007.03.013. MR2360930(2008k:35338)

[4] Adalberto P. Bergamasco, Alexandre Kirilov, Wagner Vieira Leite Nunes, and Sérgio Luís Zani, On the global solvability for overdetermined systems, Trans. Amer. Math. Soc. 364 (2012), no. 9, 4533-4549, DOI 10.1090/S0002-9947-2012-05414-6. MR2922600

[5] Adalberto P. Bergamasco, Wagner V. L. Nunes, and Sérgio Luís Zani, Global analytic hypoellipticity and pseudoperiodic functions (English, with English and Portuguese summaries), Mat. Contemp. 18 (2000), 43-57. VI Workshop on Partial Differential Equations, Part I (Rio de Janeiro, 1999). MR1812862(2001m:35062)

[6] Adalberto P. Bergamasco, Wagner V. L. Nunes, and Sérgio Luís Zani, Global properties of a class of overdetermined systems, J. Funct. Anal. 200 (2003), no. 1, 31-64, DOI 10.1016/S0022-1236(02)00055-1. MR1974087 (2004c:35295)

[7] Adalberto P. Bergamasco and Gerson Petronilho, Global solvability of a class of involutive systems, J. Math. Anal. Appl. 233 (1999), no. 1, 314-327, DOI 10.1006/jmaa.1999.6310. MR:1684389 (2001e:35002)

[8] Shiferaw Berhanu, Paulo D. Cordaro, and Jorge Hounie, An introduction to involutive structures, New Mathematical Monographs, vol. 6, Cambridge University Press, Cambridge, 2008. MR:2397326 (2009b:32048)

[9] Fernando Cardoso and Jorge Hounie, Global solvability of an abstract complex, Proc. Amer. Math. Soc. 65 (1977), no. 1, 117-124. MR0463721 (57 \#3663)

[10] François Trèves, Hypo-analytic structures, Princeton Mathematical Series, vol. 40, Princeton University Press, Princeton, NJ, 1992. Local theory. MR.1200459 (94e:35014)

Departamento de Matemática, iCMC-USP, Caixa Postal 668, 13560-970, São Carlos, SP, BRAZIL

E-mail address: apbergam@icmc.usp.br

Departamento de Matemática, UfPr, Caixa Postal 19081, 1, 81531-980, Curitiba, PR, BRASIL

E-mail address: akirilov@ufpr.br

Departamento de Matemática, ICMC-USP, Caixa Postal 668, 13560-970, São Carlos, SP, BRAZIL

E-mail address: wvlnunes@icmc.usp.br

Departamento de Matemática, ICMC-USP, Caixa Postal 668, 13560-970, São Carlos, SP, BRAZIL

E-mail address: szani@icmc.usp.br 